\title{
Editorial
}

\section{Well-Being Therapy: Current Indications and Emerging Perspectives}

\author{
Giovanni A. Fava \\ Department of Psychology, University of Bologna, Bologna, Italy; Department of Psychiatry, State University of \\ New York at Buffalo, Buffalo, N.Y., USA
}

In the 1990s, as other investigators, I was particularly concerned about the high risk of relapse in depression and its link with residual symptomatology [1]. It was not easy to make the patients better, but it was even more difficult to keep them well. My co-workers and I had performed a small controlled study on the effects of addressing residual symptomatology with cognitive-behavior therapy (CBT) in reducing relapse rates. Compared with a control condition, there were significant differences after 4 years [2], but not after 6 years [3]. I felt that what I had introduced (a sequential strategy: first treatment with antidepressant drugs and then CBT of residual symptoms) was good, but it was not sufficient.

I was looking for a psychotherapeutic strategy that could increase the level of recovery. This was the setting where I developed a psychotherapeutic technique for increasing psychological well-being, well-being therapy (WBT) [4]. Since CBT of residual symptoms of depression was found to be more effective than clinical management [5], I thought that comparing the two strategies (CBT and WBT) could be the first step. Twenty patients with mood and anxiety disorders who had been successfully treated by behavioral (anxiety disorders) or pharmacological (mood disorders) methods were randomly assigned to either WBT or CBT of residual symptoms [6]. Both WBT and CBT were associated with a significant reduction of residual symptoms, as measured by the Clin-

\section{KARGER}

E-Mail karger@karger.com

www.karger.com/pps ical Interview for Depression (CID) [7], and increases in well-being, as assessed by the Psychological Well-Being (PWB) scales [8]. However, when residual symptoms of the two groups were compared after treatment, a significant advantage of WBT over CBT was observed with the CID. WBT was also associated with a significant increase in PWB, particularly in the personal growth scale [6]. The small number of subjects suggested caution in interpreting this difference and the need for further studies with larger samples of patients with specific mood or anxiety disorders.

This is why I decided to include WBT in the treatment package, together with CBT of residual symptoms and lifestyle modification, of a study concerned with patients with a severe form of recurrent depression defined as the occurrence of 3 or more episodes of unipolar depression, with the immediately preceding episode being no more than 2.5 years before the onset of the current episode [9]. Forty patients with recurrent major depression, who had been successfully treated with antidepressant drugs, were randomly assigned to either this package including WBT or clinical management. In clinical management the same number of sessions that was used in the experimental condition was given. Clinical management consisted of reviewing the clinical status of the patient and providing the patient with support and advice, if necessary. In both groups, antidepressant drugs were tapered and discontinued. The (c) 2016 S. Karger AG, Basel

0033-3190/16/0853-0136\$39.50/0
Giovanni A. Fava, MD

Department of Psychology, University of Bologna viale Berti Pichat 5

IT-40127 Bologna (Italy)

E-Mail giovanniandrea.fava@ unibo.it 
group that received CBT and WBT had a significantly lower level of residual symptoms after drug discontinuation in comparison with the clinical management group. CBT also resulted in a significantly lower relapse rate $(25 \%)$ at a 2 -year follow-up than did clinical management $(80 \%)$. At a 6 -year follow-up [10], the relapse rate was $40 \%$ in the former group and $90 \%$ in the latter. Further, the group treated with CBT and WBT had a significantly lower number of recurrences when multiple relapses were taken into account. Even though it was a small and preliminary study, the results were quite impressive: more than half of the patients treated with CBT and WBT were well and drug free at a 6-year follow-up [10].

The findings were replicated by three independent studies. In a multi-center trial performed in Germany, 180 patients with 3 or more episodes of major depression were randomized to a combination of CBT, WBT and mindfulness-based cognitive therapy or to manualized psychoeducation [11]. Even though the follow-up was limited to 1 year (in our study the most substantial differences emerged later) and medication was continued, there was a significant effect of the experimental condition on the relapse rate of the patients with high risk of recurrence.

In the USA, Kennard et al. [12] applied the sequential treatment we had introduced in adults [9] to 144 children and adolescents with major depression. They were treated with fluoxetine for 6 weeks, and those who displayed an adequate response were randomized to receive either continued medication management or CBT to address residual symptoms and WBT in addition to fluoxetine. The CBT/WBT combination was effective in reducing the risk of relapse, a finding that was quite exceptional in the literature concerned with children and adolescents with major depression. Unfortunately, unlike our original study [9], medication was also continued in the CBT/ WBT group, despite the problems that are related to longterm treatment with antidepressant drugs in that patient population [13].

A third confirmation came from Iran in a study by Moeenizadeh and Salagame [14]. Forty high-school and university students suffering from depression were randomly assigned to WBT or CBT. The results unequivocally showed that WBT was more effective than CBT in improving symptoms of depression [14]. The severity of the depressive disturbances was not specifically evaluated and the symptomatology was probably mild. Nonetheless, the results were quite impressive.

In the course of the years WBT gained from the insights that derived from its application to other disorders
[15]. The original protocol [4] underwent a first modification in 2009 [15] and was eventually finalized in a treatment manual [16]. I will outline the main technical characteristics and aims of WBT, its currents areas of application and some emerging perspectives.

\section{The Structure}

WBT is a short-term psychotherapeutic strategy that emphasizes self-observation, with the use of a structured diary, interaction between patients and therapists and homework. WBT is based on a model of psychological well-being that was originally developed by Marie Jahoda in 1958 [17]. She outlined 6 criteria for positive mental health: autonomy (regulation of behavior from within), environmental mastery, satisfactory interactions with other people and the milieu, the individual's style and degree of growth, development or self-actualization, the attitudes of an individual toward his/her own self (self-perception/acceptance) and the individual's balance and integration of psychic forces. Carol Ryff further elaborated the first 5 dimensions of positive functioning and introduced a method for their assessment, the PWB scales [7]. While initially WBT was simply aimed at increasing psychological well-being, its goal was subsequently refined in the achievement of a state of euthymia, Jahoda's sixth criterion [17]. She defined it as the individual's balance of psychic forces (flexibility), a unifying outlook on life which guides actions and feelings for shaping the future accordingly, and resistance to stress (resilience and anxiety or frustration tolerance). It is not simply generic (and clinically useless) advice of avoiding excesses and extremes; it is how the individual adjusts the psychological dimensions of well-being to changing needs [18].

\section{Articulation}

WBT may be used as the only therapeutic strategy. In this case the number of sessions may range from 8 to $16-$ 20. The duration of each session may range from 45 to 60 min. WBT may also be used in sequential combination with other psychotherapeutic strategies, in particular $\mathrm{CBT}$, and in this case the number of sessions may be abridged to 4-6 [16]. The sequential combination of CBT/WBT has characterized its use so far [16].

The initial phase is concerned with self-observation of psychological well-being. Once the instances of well-being are properly recognized, the patient is encouraged to identify thoughts, beliefs and behaviors leading to premature interruption of well-being (intermediate phase). The 
final part involves cognitive restructuring of dysfunctional dimensions of psychological well-being and meeting the challenge that optimal experiences may entail [16].

WBT was originally conceived as an individual therapy [4], but it may be amenable to a group format [14]. This modality may increase sharing optimal experiences and personal meanings of psychological well-being. It is also conceivable, even though yet to be tested, that WBT interventions may increase the effectiveness of couple and family interventions. Kauffman and Silberman [19] have illustrated adaptations of positive psychology interventions that may improve the outcomes of couple therapy. Fostering the positive in relationships is indeed a target of many family and couple approaches and elements of WBT may facilitate such a process.

An additional modality for WBT involves the growing area of computer-assisted methods. Treatment programs or mobile applications could help clinicians and reach a wider audience.

\section{Characteristic Features}

Within the broad and highly heterogeneous spectrum of positive interventions [20], WBT stands for some specific aspects:

Monitoring of Psychological Well-Being in a Diary. Patients are encouraged to identify episodes of well-being and to set them into a situational context. They are asked to report in a structured diary the circumstances surrounding their episodes of well-being, rated on a scale of $0-100$, with 0 being absence of well-being and 100 the most intense well-being that could be experienced. Such a search also involves optimal experiences. These are characterized by the perception of high environmental challenges and environmental mastery, deep concentration, involvement, enjoyment, control of the situation, clear feedback on the course of activity and intrinsic motivation [21].

Identification of Low Tolerance to Well-Being by Seeking Automatic Thoughts. Once the instances of well-being are properly recognized, the patient is encouraged to identify thoughts and beliefs leading to premature interruption of well-being (automatic thoughts), as is performed in cognitive therapy [22]. The trigger for self-observation is, however, different, being based on well-being instead of distress, as illustrated in figures 1 and 2.

Behavioral Exposure. The therapist may also reinforce and encourage activities that are likely to elicit well-being and optimal experiences (for instance, assigning the task of undertaking particular pleasurable activities for a certain time each day). Such reinforcement may also result

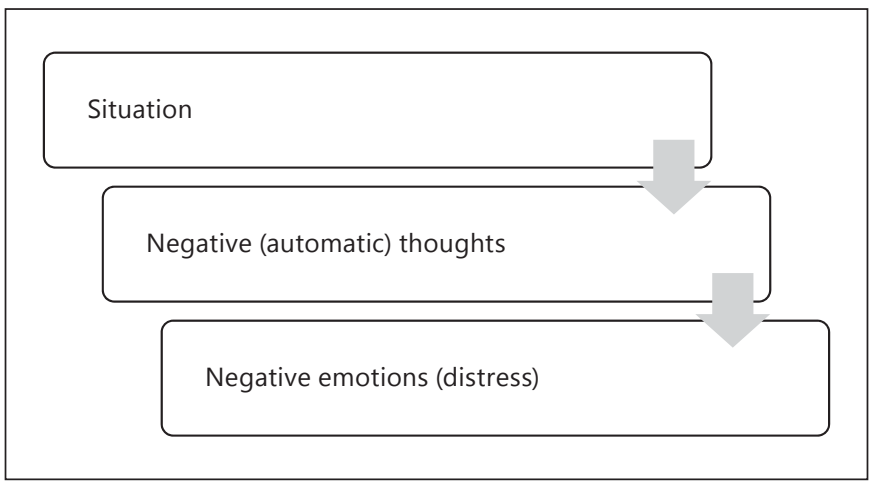

Fig. 1. Basic mechanism of cognitive therapy (from Well-Being Therapy. Treatment Manual and Clinical Applications, by Fava [16]).

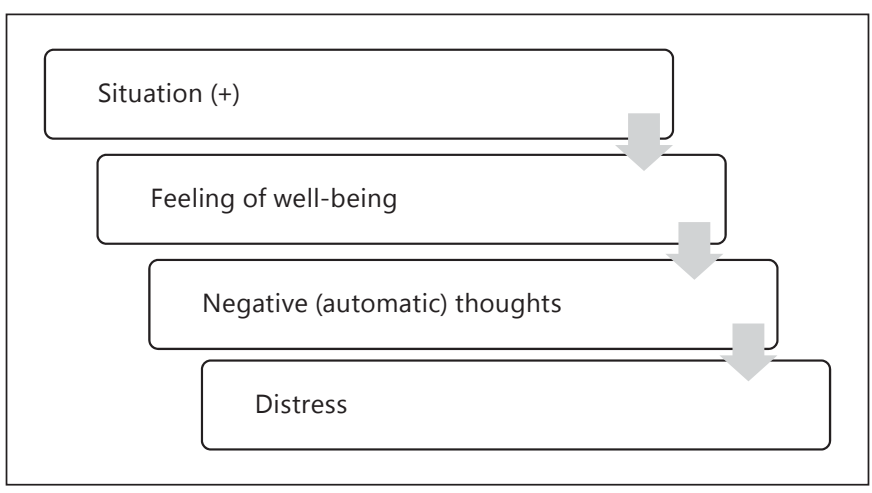

Fig. 2. Basic mechanism of well-being therapy (from Well-Being Therapy. Treatment Manual and Clinical Applications, by Fava [16]).

in graded task assignments [22], with special reference to exposure to feared or challenging situations which the patient is likely to avoid. Meeting the challenge that optimal experiences may entail is emphasized, because it is through this challenge that growth and improvement of self can take place.

Cognitive Restructuring Using Specific Psychological Well-Being Models. The monitoring of the course of episodes of well-being allows the therapist to realize specific impairments or excessive levels in well-being dimensions according to the conceptual framework of Jahoda [17] and Ryff [7]. For example, the therapist could explain that autonomy consists of possessing an internal locus of control, independence and self-determination, or that personal growth consists of being open to new experiences and considering the self as expanding over time, if the patient's attitudes show impairments in these specific ar- 
eas. The patient thus becomes able to readily identify moments of well-being, be aware of interruptions to wellbeing feelings (cognitions), utilize cognitive behavioral techniques to address these interruptions and pursue optimal experiences.

Individualized and Balanced Focus. Patients are not simply encouraged to pursue the highest possible levels in psychological well-being in all dimensions, as is found to be the case in most positive interventions [20], but to obtain a balanced functioning, subsumed under the rubric of euthymia [18]. Positive characteristics such as gratitude and autonomy often exist on a continuum [23]. They are neither 'negative' nor 'positive': their impact depends on the specific situation and on the interaction with concurrent distress and other psychological attitudes. This optimal-balanced well-being could be different from patient to patient, according to factors such as personality traits, social roles and cultural and social contexts.

\section{Current Indications}

WBT has been tested in a number of controlled trials, mostly as an adjunctive treatment ingredient. Unlike many other psychotherapeutic strategies, it was not conceived as a cure for mental disorders, but as a therapeutic tool to be incorporated in a therapeutic plan. As a general indication, it is difficult to apply WBT as the first-line treatment of an acute psychiatric disorder. It may be more suitable for second- or third-line treatments. Most of the patients who are seen in clinical practice have complex and chronic disorders [24]. It is simply wishful thinking to believe that one course of treatment will be sufficient for yielding lasting and satisfactory remission. Further, WBT was not conceived to be used in every patient who meets specific diagnostic criteria [16]. It should follow clinical reasoning and case formulation facilitated by the use of macro-analysis [25]. This latter consists of establishing a relationship between co-occurring syndromes and problems. Macroanalysis is not limited to diagnostic entities, as is the case in the concept of comorbidity in DSM, but also to problems that are judged by the clinician to affect a person's life. Macro-analysis starts from the assumption that, in most cases, there are functional relationships among different problem areas and that the targets of treatment may vary during the course of the disturbances. Different lines of treatment may be chosen at different times. The hierarchical organization that is chosen may depend on a variety of factors (urgency, availability of treatment tools, priorities established by the clinician and/or the patient, etc.) [24,
25]. Staging is another key determinant of its use, both in terms of longitudinal development of a disorder and treatment response [26]. Not surprisingly, the three main current indications of WBT are transdiagnostic.

\section{Increasing the Level of Recovery}

The sequential combination of CBT and WBT in recurrent depression has resulted in a decreased rate of relapse [10-12]. However, this is a general characteristic of trials using the sequential model that consists of pharmacotherapy for addressing the acute symptoms of depression and psychotherapy in its residual phase [27, 28]. From the available studies [10-12] we have no way of knowing whether WBT was a specific effective ingredient and what the mechanism was in decreasing the likelihood of relapse. A dismantling study that was performed in generalized anxiety disorder [29] suggested that an increased level of recovery could indeed be obtained with the addition of WBT to CBT. Twenty patients were randomly assigned to 8 sessions of CBT or the sequential administration of CBT followed by another 4 sessions of WBT. Both treatments were associated with a significant reduction of anxiety. However, significant advantages of the CBT/WBT sequential combination over CBT were observed, both in terms of symptom reduction and psychological well-being improvement as measured by the CID [8] and the PWB [7]. These results suggested the feasibility and clinical advantages of adding WBT to the treatment of generalized anxiety disorder. The decrease in anxiety, as measured by the CID [8], might be due to the fact that self-monitoring of episodes of well-being may lead to a more comprehensive identification of automatic thoughts than that entailed by the customary monitoring of episodes of distress in cognitive therapy and may thus result in more effective cognitive restructuring. An increase in psychological wellbeing, as measured by the PWB [7], occurred also with CBT only, but not to the degree that took place with WBT, suggesting a specific mechanism for WBT. Both mechanisms indicate a role for WBT in increasing the level of recovery. While the clinical benefits have been substantiated in depression and generalized anxiety disorder, this appears to be a target for a number of other mental disorders. For instance, Penn et al. [30] have postulated a role for WBT in improving functional outcomes as an additional ingredient to CBT in psychotic disorders. Indeed, subjective well-being appears to be impaired in schizophrenia and is associated with reduced anterior cingulated activity during reward processing, which may induce reduced integration of environmental stimuli, motivated behavior and reward outcome [31]. 
Table 1. Definition of euthymia (from Fava and Bech [18])

A state of euthymia is characterized by the following features:

a Lack of mood disturbances that can be subsumed under diagnostic rubrics; if the subject has a prior history of mood disorder, he/she should be in full remission; if sadness, anxiety or irritable mood are experienced, they tend to be short-lived, related to specific situations and do not significantly affect everyday life

b The subject feels cheerful, calm, active, interested in things and sleep is refreshing or restorative

c The subject displays balance and integration of psychic forces (flexibility), a unifying outlook on life which guides actions and feelings for shaping future accordingly, and resistance to stress (resilience and anxiety or frustration tolerance)

\section{Modulating Mood}

WBT was applied to the treatment of cyclothymic disorder, which involves mild or moderate fluctuations of mood, thought and behavior without meeting formal diagnostic criteria for either major depressive disorder or mania [32]. It is a common and disabling condition that does not attract much research attention since no drugs have been patented for its treatment. Sixty-two patients with cyclothymic disorder were randomly assigned to the sequential combination of CBT and WBT or clinical management. An independent blinded evaluator assessed the patients before treatment, after therapy and at 1- and 2-year follow-ups. The CID [8] and the Mania Scale [33] were used to evaluate symptoms. At posttreatment evaluation, significant differences were found in outcome measures, with greater improvements in the CBT/WBT group compared with clinical management. Therapeutic gains were maintained at 1- and 2-year follow-ups [32]. The results thus indicated that WBT may address both polarities of mood swings and is geared to a state of euthymia, as outlined in table 1. In 1991, Garamoni et al. [34] suggested that healthy functioning is characterized by an optimal balance of positive and negative cognitions or affects, and that psychopathology is marked by deviations from the optimal balance. As pointed out by Wood and Tarrier [23], excessively elevated levels of positive emotions can become detrimental and are more connected with mental disorders and impaired functioning. Can the WBT target of euthymia decrease vulnerability to depression in anxiety disturbances? Why do many patients in the longitudinal course of anxiety disorders develop depression and other patients do not [18]?

\section{Educational Purposes}

Three randomized controlled trials in educational settings indicated that protocols based on WBT may be suitable for promoting mechanisms of resilience and psychological well-being [35-37]. In the first pilot study, school interventions (4 class sessions lasting a couple of hours) were performed in a population of 111 middleschool students randomly assigned to either a protocol using theories and techniques derived from CBT or a protocol derived from WBT. Both school-based interventions resulted in a comparable improvement in symptoms and psychological well-being [35]. This pilot investigation suggested that well-being-enhancing strategies could match CBT in the prevention of psychological distress and in promoting optimal human functioning among children. The differential effects of WBT and CBT approaches have been subsequently explored in another controlled school intervention, involving more sessions and an adequate follow-up [36]. In this trial, 162 students attending middle schools were randomly assigned to either a protocol derived from WBT or an anxiety management protocol. The results of this investigation showed that WBT was found to produce significant improvements in the autonomy scale of PWB [7] and in the friendliness scale of the Symptom Questionnaire [38], whereas anxiety management ameliorated anxiety only.

WBT school interventions were extended to highschool students, who are considered to be a more 'at risk' population for mood and anxiety disorders [39]. School interventions were performed in a sample of 227 students [37]. The classes were randomly assigned to either a protocol derived from WBT or an attentionplacebo protocol, which consisted of relaxation techniques, group discussion of common problems reported by students and conflict resolution. The WBT intervention was found to be significantly more effective in promoting psychological well-being, with particular reference to personal growth, compared with the attention-placebo protocol. Further, it was found to be more effective in decreasing distress, in particular anxiety and somatization. The beneficial effects of the WBT protocol in decreasing anxiety and somatization were maintained at the follow-up, whereas in the attention-placebo group improvements faded and disappeared [37]. The results thus indicated that WBT in educational settings may yield enduring results in terms of positive emotions and psychological well-being. Each session was conducted by two psychologists in the presence of the teacher. 
There is little doubt that WBT may display great potential with children and adolescents. The main reason is the high flexibility that characterizes this age population, which lends itself to the achievement of new balances in the terms of Jahoda [17]. This potential may be extended from clinical to educational settings [39].

\section{Emerging Perspectives}

A potential role for WBT can be postulated and deserves to be tested in a number of clinical conditions.

\section{Treatment Resistance}

WBT may be of particular value in the high proportion of patients who fail to respond to standard pharmacological and/or psychotherapeutic treatments. Six patients with agoraphobia and panic attacks who completed a controlled trial with a cross-over design, encompassing three treatment modalities, namely exposure alone, cognitive therapy with exposure and exposure associated with imipramine [40], but still suffered from panic attacks, were offered a course of WBT. Three patients accepted. WBT was associated with the prolongation of exposure in vivo homework [4]. Of the 3 patients, 2 achieved panic-free status. It is obviously very difficult to draw conclusions from this very small trial, which involved only half of the patients who still suffered from panic disorder. A placebo (nonspecific) effect is possible, even though unlikely in patients who had unsuccessfully undergone three consecutive trials. Since the controlled trial had disclosed a significant effect of the time factor [40], the results might have been simply due to prolongation of exposure. However, it is also possible that WBT helped the 2 patients undergoing exposure and increased their compliance as to exposure homework. This, indeed, appeared to have been improved according to the therapist's ratings [40]. Cosci [41] described the case of a patient with panic disorder, agoraphobia and a major depressive episode who failed to respond to paroxetine and CBT and successfully responded to WBT. This patient was unable to identify automatic thoughts by monitoring distress with cognitive therapy, whereas she was able to do so while monitoring well-being with WBT. Interestingly, after WBT she was able to complete cognitive therapy [41].

WBT has been applied to the case of a depressed patient who displayed treatment resistance. She had responded to an antidepressant drug well; the medication was then tapered and discontinued [42]. Three months later depression came back; this time, however, the same medication that was given the first time did not work. After some pharmacological attempts, the sequential combination of CBT and WBT was attempted. She got better and stayed well. 'I now have a 12-year follow-up with no relapses and psychotropic medications'. Interestingly, since the mechanisms of oppositional tolerance with antidepressant drugs may involve the HPA axis [42], we also measured 24-hour urinary free cortisol which provides an overall assessment of the production of cortisol. The HPA axis was normalized by CBT/WBT [42]. Future research may disclose whether WBT has a place in treatment resistance in depression. Indeed, 2 other interesting cases were reported in the Netherlands [43]. One involved a patient with depression that was refractory to both pharmacological treatment and CBT and was successfully treated with WBT. In the other case, WBT was applied to a patient with DSM dysthymia and yielded remission [43].

\section{Overcoming Trauma and Increasing Resilience}

Two cases have been reported on the use of WBT alone or in sequential combination with exposure for overcoming posttraumatic stress disorder, with the central trauma being discussed only in the initial historytaking session [44]. Research on the neurobiological correlates of resilience has disclosed how different neural circuits (reward, fear conditioning and extinction, social behavior) may involve the same brain structures, particularly the amygdala, the nucleus accumbens and the medial prefrontal cortex [45]. Reconsolidation is a process in which old, reactivated memories undergo consolidation: each time a traumatic memory is retrieved, it is integrated into an ongoing perceptual and emotional experience, which involves NMDA (N-methyl-D-aspartate) and $\beta$-adrenergic receptors and requires cAMP (cyclic adenosine monophosphate) response element binding protein induction [45]. Singer et al. [46], on the basis of preclinical evidence, suggested that WBT may stimulate dendrite networks in the hippocampus and induce spine retraction in the basolateral amygdala (a site of storage for memories of fearful or stressful experiences), leading to a weakening of distress and traumatic memories.

There has been growing awareness of the fact that traumatic experiences can also give rise to positive transformations, subsumed under the rubric of posttraumatic growth [47]. Positive changes can be observed in self-concept (e.g. new evaluation of one's strength and resilience), appreciation of new possibilities in life, social relations, 
Table 2. The Mental Pain Questionnaire (MPQ) (from Fava [16])

Mental or psychological pain is an experience that is part of life. It is different from physical pain. We would like to learn about your experience of mental pain in the past week. There is no right or wrong answer. Please work quickly.

1 I feel pain

2 My heart is broken

3 I will never find again what I have lost

4 My pain is everywhere

5 My pain is with me all the time

6 I cannot understand why I feel this pain

7 I feel empty

8 My life makes no sense

9 My pain will never go away

10 The only way to stop my pain is to die

$\begin{array}{ll}\text { yes } & \text { no } \\ \text { yes } & \text { no } \\ \text { yes } & \text { no } \\ \text { yes } & \text { no } \\ \text { yes } & \text { no } \\ \text { true } & \text { false } \\ \text { yes } & \text { no } \\ \text { true } & \text { false } \\ \text { true } & \text { false } \\ \text { true } & \text { false }\end{array}$

Scoring for each item yes $/$ true $=1 ;$ no/false $=0$; total score range: $0-10$.

hierarchy of values and priorities, and spiritual growth. WBT may be uniquely suited for facilitating the process of posttraumatic growth.

\section{Mental Pain}

An issue that is not sufficiently appreciated is the experience of mental pain many patients have [48-51]. Patients sometimes mention this experience spontaneously, but in other cases only upon specific questions (which, however, are seldom asked). Mental pain may be worse than most forms of physical pain, because it is not localized and often has no apparent reason. Table 2 indicates some characteristics of mental pain, as depicted in a selfrating scale. Grief provides an example of the sense of emptiness, loss of meaning and suffering that mental pain entails. Mental pain may or may not be associated with anxiety and/or depression [48-51]. It is conceivable and yet to be tested that WBT may counteract the manifestations of mental pain [16]. Since positive affects have been found to attenuate both the perception of physical pain and its affective responses [52], WBT may also entail favorable effects in the case of physical pain.

\section{Discontinuing Psychotropic Drugs}

Psychotropic drug treatment, particularly when it is protracted in time, may cause various forms of dependence. Withdrawal symptoms do not necessarily wane after drug discontinuation and may build into a persistent postwithdrawal disorder [53]. These symptoms may constitute an iatrogenic comorbidity that affects the course of illness and the response to subsequent treatments [13]. The discontinuation of antidepressant drugs such as selective serotonin reuptake inhibitors, duloxetine and venlafaxine constitutes a major clinical challenge [53]. A protocol based on the sequential combination of CBT and WBT has been devised and tested in case reports [54]. The addition of WBT, which has also been suggested in substance dependence [55], had the specific function of counteracting the effects of pharmaceutical propaganda on patients. Such effects were remarkably anticipated by Lipowski [56] more than 25 years ago.

'Another current fad is to tell patients that they suffer from a chemical imbalance in the brain. The explanatory power of this statement is of about the same order as if you said to the patient: "you are alive". It confuses the distinction between etiology and correlation, and cause and mechanism, a common confusion in our field. It gives the patient a misleading impression that his or her imbalance is the cause of his or her illness, that it needs to be fixed by purely chemical means, that psychotherapy is useless, and that personal efforts and responsibility have no part to play in getting better' [56, p. 252].

It is important to convey the message that 'there is life after antidepressant drugs' and WBT may be a helpful tool in this freedom path. When the catastrophic iatrogenic effects of the inappropriate use of antidepressant drugs, particularly in anxiety disorders, is able to emerge from a very tight censorship [57], the importance of discontinuation strategies will then be fully appreciated [53, 57].

\section{Improving Medical Outcomes}

The need to include the consideration of psychosocial factors (functioning in daily life, psychiatric and psychological symptoms, quality of life, illness behavior) has emerged as a crucial part of investigation and patient care [58]. These aspects have become particularly important in chronic diseases, where cure cannot take place, and also extend to family caregivers of chronically ill patients and health providers [58]. It is thus conceivable to postulate a role for WBT in the setting of medical disease, to counteract the limitations and challenges induced by illness experience. A randomized controlled trial, headed by Chiara Rafanelli [in preparation], on addressing depressive symptoms and demoralization after myocardial infarction is currently in progress. Patients are being randomized to CBT/WBT sequential combination or clinical management. In some way, what Chiara Rafanelli is attempting to do with patients who suffered from a myocardial infarction pertains to rehabilitation medicine, 
which is another important potential area of application of WBT. The process of rehabilitation, in fact, requires the persuit of well-being and changes in lifestyle as primary targets of intervention [58].

The use of WBT in the setting of medical disease may not simply be that of promoting psychological well-being and coping in the face of serious illness [59]. In recent years there has been increasing evidence suggesting that stressful conditions may elicit a pattern of CTRA (conserved transcriptional response to adversity), in which there is an increased expression of proinflammatory genes and a concurrent decreased expression of type 1 interferon innate antiviral response and IgG antibody synthesis [60]. Such patterns have been implicated in the pathophysiology of cancer [61] and cardiovascular diseases [62]. Frederickson et al. [63] showed that individuals with high psychological well-being presented reduced CTRA gene expression, which introduces a potential protective role of WBT in a number of medical disorders.

\section{Improving Health Attitudes and Behavior}

An unhealthy lifestyle is a major risk factor for many of the most prevalent diseases, such as diabetes, obesity and cardiovascular illness [58]. Switching the general population to healthy lifestyles is a major source of prevention, particularly in the younger population. In adolescents, decreased well-being has been associated with unhealthy behavior, such as smoking, physical inactivity and insufficient sleep [64]. WBT may thus contribute to improve health attitudes and behavior. The American Academy of Pediatrics has underscored the need to address the current epidemic of childhood obesity through enhanced adherence to dietary guidelines and increasing physical activity [65]. Significant impairments in psychological well-being in patients with eating disorders compared with healthy controls have been recently documented [66]. These findings may pave the way for assessing the value of WBT in eating disorders. WBT may particularly address body image disturbances, whether associated with eating disorders or not $[67,68]$.

\section{Conclusions}

The studies that are summarized indicate that the potential role of WBT is broader than that originally assumed, that is, decreasing the risk of relapse in the residual phase of mood and anxiety disorders [69]. Its updated scope encompasses increasing resilience in a variety of psychiatric and medical conditions, modulating psychological well-being and mood, and developing alternative pathways to established treatment modalities, including psychotropic drugs. An important characteristic of WBT is self-observation of psychological well-being associated with specific homework. Such a perspective is different from interventions that are labeled as positive but are actually distress oriented. Another important feature of WBT is the assumption that imbalances in well-being and distress may vary from one illness to another and from patient to patient [70]. The pursuit of euthymia [18] can thus only be achieved with a personalized approach that characterizes the treatment protocol and requires a comprehensive initial evaluation. The manualization of WBT [16] may facilitate its individualized application, and the insights gained by clinicians and investigators may refine its current use and indications. The model is realistic, instead of idealistic, and may be frustrating to psychotherapists who are in need of fixed conceptual schemas, but it is in keeping with the complexity of clinical situations that are encountered in everyday practice.

\section{Acknowledgment}

Drs Fiammetta Cosci, Jenny Guidi, Emanuela Offidani, Chiara Rafanelli, Nicoletta Sonino and Elena Tomba provided helpful criticism and comments.

\section{Disclosure Statement}

The author has written a book on WBT [16], for which he receives no royalties. He has no financial conflicts of interest to declare.
References

Well-Being Therapy
Psychother Psychosom 2016;85:136-145 DOI: $10.1159 / 000444114$

\footnotetext{
3 Fava GA, Ruini C, Rafanelli C, Finos L, Conti S, Grandi S: Six-year outcome of cognitive behavior therapy for prevention of recurrent depression. Am J Psychiatry 1998;161:18721876.

4 Fava GA: Well-being therapy: conceptual and
} tive behavioral treatment of residual symp- 4 Fava GA: Well-being therapy: conceptual and
toms in major depression. Am J Psychiatry
technical issues. Psychother Psychosom 1999; $\begin{array}{ll}\text { tive behavioral treatment of residual symp- } & \text { Fava GA: Well-being therapy: conceptual and } \\ \text { toms in major depression. Am J Psychiatry } & \text { technical issues. Psychother Psychosom 1999; } \\ \text { 1996;153:945-947. } & 68: 171-179 .\end{array}$ 1996;153:945-947.

Fava GA: The concept of recovery in affective disorders. Psychother Psychosom 1996;65

Fava GA, Grandi S, Zielezny M, Rafanelli C, Canestrari R: Four-year outcome for cogni- 
5 Fava GA, Grandi S, Zielezny M, Canestrari R, Morphy MA: Cognitive-behavioral treatment of residual symptoms in primary major depressive disorder. Am J Psychiatry 1994;151: 1295-1299.

-6 Fava GA, Rafanelli C, Cazzaro M, Conti S, Grandi S: Well-being therapy: a novel psychotherapeutic approach for residual symptoms of affective disorders. Psychol Med 1998;28: 475-480.

7 Ryff CD: Psychological well-being revisited. Psychother Psychosom 2014;83:10-28.

8 Guidi J, Fava GA, Bech P, Paykel ES: The Clinical Interview for Depression: a comprehensive review of studies and clinimetric properties. Psychother Psychosom 2011;80:10-27.

-9 Fava GA, Rafanelli C, Grandi S, Conti S, Belluardo P: Prevention of recurrent depression with cognitive behavioral therapy: preliminary findings. Arch Gen Psychiatry 1998;55: 816-820.

10 Fava GA, Ruini C, Rafanelli C, Finos L, Conti $S$, Grandi S: Six-year outcome of cognitive behavior therapy for prevention of recurrent depression. Am J Psychiatry 2004;161:18721876.

-11 Stangier U, Hilling C, Heidenreich T, Risch AK, Barocka A, Schlösser R, Kronfeld K, Ruckes C, Berger H, Röschke J, Weck F, Volk S, Hambrecht M, Serfling R, Erkwoh R, Stirn A, Sobanski T, Hautzinger M: Maintenance cognitive-behavioral therapy and manualized psychoeducation in the treatment of recurrent depression: a multicenter prospective randomized controlled study. Am J Psychiatry 2013;170:624-632.

$\checkmark 12$ Kennard BD, Emslie GJ, Mayes TL, Nakonezny PA, Jones JM, Foxwell AA, King J: Sequential treatment with fluoxetine and relapse-prevention CBT to improve outcomes in pediatric depression. Am J Psychiatry 2014; 171:1083-1090.

13 Offidani E, Fava GA, Sonino N: Iatrogenic comorbidity in childhood and adolescence: new insights from the use of antidepressant drugs. CNS Drugs 2014;28:769-774.

14 Moeenizadeh M, Salagame KK: The impact of well-being therapy on symptoms of depression. Int J Psychol Stud 2010;2:223-230.

15 Fava GA, Tomba E: Increasing psychological well-being and resilience by psychotherapeutic methods. J Pers 2009;77:1902-1934.

16 Fava GA: Well-Being Therapy. Treatment Manual and Clinical Applications. Basel, Karger, 2016.

17 Jahoda M: Current Concepts of Positive Mental Health. New York, Basic Books, 1958.

18 Fava GA, Bech P: The concept of euthymia. Psychother Psychosom 2016;85:1-5.

19 Kauffman C, Silberman J: Finding and fostering the positive in relationships: positive interventions in couples therapy. J Clin Psychol 2009;65:520-531.

20 Quoidbach J, Mikolajczak M, Gross JJ: Positive interventions: an emotion regulation perspective. Psychol Bull 2015;141:655-693.
21 Csikszentmihalyi M, Csikszentmihalyi I: Optimal Experience. Psychological Studies of Flow in Consciousness. New York, Cambridge University Press, 1988.

22 Beck AT, Rush AJ, Shaw BF, Emery G: Cognitive Therapy of Depression. New York, Guilford Press, 1979.

23 Wood AM, Tarrier N: Positive clinical psychology: a new vision and strategy for integrated research and practice. Clin Psychol Rev 2010;30:819-829.

24 Fava GA, Rafanelli C, Tomba E: The clinical process in psychiatry. J Clin Psychiatry 2012; 73:177-184.

25 Emmelkamp PMG, Bouman TK, Scholing A: Anxiety Disorders. Chichester, Wiley, 1993.

26 Cosci F, Fava GA: Staging of mental disorders: systematic review. Psychother Psychosom 2013;82:20-34.

27 Fava GA: Sequential treatment. Psychother Psychosom 1999;68:227-229.

28 Guidi J, Tomba E, Fava GA: The sequential integration of pharmacotherapy and psychotherapy in the treatment of major depressive disorder: a meta-analysis of the sequential model and a critical review of the literature. Am J Psychiatry 2016;173:128-137.

29 Fava GA, Ruini C, Rafanelli C, Finos L, Salmaso L, Mangelli L, Sirigatti S: Well-being therapy of generalized anxiety disorder. Psychother Psychosom 2005;74:26-30.

30 Penn DL, Mueser KT, Tarrier N, Gloege A, Cather C, Serrano D, Otto MN: Supportive therapy for schizophrenia. Schizophr Bull 2004;30:101-112.

31 Gilleen J, Shergill SS, Kapur S: Impaired subjective well-being in schizophrenia is associated with reduced anterior cingulated activity during reward processing. Psychol Med 2015; 45:589-600.

-32 Fava GA, Rafanelli C, Tomba E, Guidi J, Grandi S: The sequential combination of cognitive behavioral treatment and well-being therapy in cyclothymic disorder. Psychother Psychosom 2011;80:136-143.

33 Bech P, Kastrup M, Rafaelsen OJ: Mini-compendium of rating scales for states of anxiety, depression, mania, schizophrenia with corresponding DSM-III syndromes. Acta Psychiatr Scand Suppl 1986;326:1-37.

34 Garamoni GL, Reynolds CF III, Thase ME: The balance of positive and negative affects in major depression: a further test of the States of Mind model. Psychiatry Res 1991;39:99108.

35 Ruini C, Belaise C, Brombin C, Caffo E, Fava GA: Well-being therapy in school settings: a pilot study. Psychother Psychosom 2006;75: 331-336.

36 Tomba E, Belaise C, Ottolini F, Ruini C, Bravi A, Albieri E, Rafanelli C, Caffo E, Fava GA: Differential effects of well-being promoting and anxiety-management strategies in a nonclinical school setting. J Anxiety Disord 2010; 24:326-333.
37 Ruini C, Ottolini, F., Tomba E, Belaise C, Albieri E, Visani D, Offidani E, Caffo E, Fava GA: School intervention for promoting psychological well-being in adolescence. J Behav Ther Exp Psychiatry 2009;40:522-532.

38 Kellner R: A symptom questionnaire. J Clin Psychiatry 1987;48:268-274.

39 Fava GA, Ruini C (eds): Increasing Psychological Well-being in Clinical and Educational Settings. Dordrecht, Springer, 2014

40 Fava GA, Savron G, Zielezny M, Grandi S, Rafanelli C, Conti S: Overcoming resistance to exposure in panic disorder with agoraphobia. Acta Psychiatr Scand 1997;95:306-312.

41 Cosci F: Well-being therapy in a patient with panic disorder who failed to respond to paroxetine and cognitive behavior therapy. Psychother Psychosom 2015;84:318-319.

42 Sonino N, Fava GA: Tolerance to antidepressant treatment may be overcome by ketoconazole. J Psychiatr Res 2003;37:171-173.

43 Meulenbeek P, Christenhusz L, Bohlmeijer E: Well-being therapy in the Netherlands. Psychother Psychosom 2015;84:316-317.

44 Belaise C, Fava GA, Marks IM: Alternatives to debriefing and modifications to cognitive behavior therapy for post-traumatic stress disorder. Psychother Psychosom 2005;74:212217.

45 Charney DS: Psychobiological mechanisms of resilience and vulnerability. Am J Psychiatry 2004;161:195-216.

46 Singer B, Friedman E, Seeman T, Fava GA, Ryff CD: Protective environments and health status. Neurobiol Aging 2005;265:s113-s118.

47 Vazquez C, Pérez-Sales P, Ochoa C: Posttraumatic growth; in Fava GA, Ruini C (eds): Increasing Psychological Well-Being in Clinical and Educational Settings. Dordrecht, Springer, pp 57-74.

48 Tossani E: The concept of mental pain. Psychother Psychosom 2013;82:67-73.

49 Meerwijk EL, Weiss SJ: Toward a unifying definition: response to 'The concept of mental pain'. Psychother Psychosom 2014;83:62-63.

-50 Blasco-Fontecilla H, Baca-Garcia E, Courtet P, Garcia Nieto R, de Leon J: Horror vacui emptiness might distinguish between major suicide repeaters and nonmajor suicide repeaters. Psychother Psychosom 2015;84:117-119.

51 de Leon J, Baca-Garcia E, Blasco-Fontecilla H: From the serotonin model of suicide to a mental pain model of suicide. Psychother Psychosom 2015;84:323-329.

52 Finan PH, Garland EL: The role of positive affect in pain and its treatment. Clin J Pain 2015;31:177-187.

53 Chouinard G, Chouinard VA: New classification of selective serotonin reuptake inhibitor (SSRI) withdrawal. Psychother Psychosom 2015;84:63-71.

54 Belaise C, Gatti A, Chouinard V-A, Chouinard G: Persistent postwithdrawal disorders induced by paroxetine, a selective serotonin reuptake inhibitor, and treated with specific cognitive behavioral therapy. Psychother Psychosom 2014;83:247-248. 
-55 Chopin MV, Peretti CS, Gisquet-Verrier P, Hoffman C, Belaid A, Chouinard G: Cocaine use disorder treated with specific cognitive behavioral therapy and adjunctive propranolol. Psychother Psychosom 2016;85:61-63.

56 Lipowski ZJ: Psychiatry: mindless or brain less, both or neither? Can J Psychiatry 1989; 35:249-254.

57 Fava GA: Rational use of antidepressant drugs. Psychother Psychosom 2014;83:197204.

58 Fava GA, Sonino N: Psychosomatic medicine. Int J Clin Pract 2010;64:1155-1161.

59 Folkman S, Greer S: Promoting psychological well-being in the face of serious illness. Psychooncology 2000;9:11-19.

60 Cole SW: Human social genomics. PLoS Genet 2014;10:e1004601.

61 Currier MB, Nemeroff CB: Depression as a risk factor for cancer. Annu Rev Med 2014;65: 203-221.
62 Nemeroff CB, Goldschmidt-Clermont PJ: Heartache and heartbreak - the link between depression and cardiovascular disease. Nat Rev Cardiol 2012;9:526-539.

63 Frederickson BL, Grewen KM, Algoes SB, Firestine AM, Arevalo JM, Ma J, Cole SW: Psychological well-being and the human conserved transcriptional response to adversity. PLoS One 2015; 10:e121839.

64 Sirri L, Ricci Garotti MG, Grandi S, Tossani E: Adolescents' hypochondriacal fears and beliefs. J Psychosom Res 2015;79:259-264.

65 Daniels SR, Greer FR; Committee on Nutrition: Lipid screening and cardiovascular health in childhood. Pediatrics 2008;122:198208.
66 Tomba E, Offidani E, Tecuta L, Schumann R, Ballardini D: Psychological well-being in outpatients with eating disorders. Int J Eat Disord 2014;47:252-258.

67 Phillips KA: Body dysmorphic disorder: common, severe and in need of treatment research. Psychother Psychosom 2014;83:325329.

68 Veale D, Anson M, Miles S, Pieta M, Costa A, Ellison N: Efficacy of cognitive behaviour therapy versus anxiety management for body dysmorphic disorder. Psychother Psychosom 2014;83:341-353.

69 Rafanelli C, Park SK, Fava GA: New psychotherapeutic approaches to residual symptoms and relapse prevention in unipolar depression. Clin Psychol Psychother 1999;6:194201

-70 Rafanelli C, Park SK, Ruini C, Ottolini F, Cazzaro M, Fava GA: Rating well-being and distress. Stress Med 2000;72:268-275. 